\title{
Topical anaesthesia for children's lacerations: an acceptable approach?
}

\author{
J M Kendall, A Charters, S E McCabe
}

\begin{abstract}
Objective-To compare the anaesthetic properties of conventional intradermal $1 \%$ plain lignocaine with a topical gel preparation of adrenaline $(1: 2000)$ and cocaine $(4 \cdot 7 \%)$ for use in treatment of children's lacerations.

Methods-Children aged 3-16 years with lacerations (not of the digits or mucous membranes) were consecutively assigned to receive either adrenaline and cocaine (AC) or lignocaine. Pain scores, as perceived by patients, parents, and staff, were measured conventionally using Wong Baker faces and visual analogue scales on administration of the local anaesthetic and on suturing the wound in the AC group $(n=56)$ and the lignocaine group $(\mathrm{n}=51)$.

Results-Mean and median pain scores on administration of the anaesthetic in the AC group were significantly lower than in the lignocaine group as perceived by patient $(P<0 \cdot 001)$, parent $(P<0.001)$, and staff $(P<0 \cdot 001)$. There was no significant difference in mean and median pain scores between the two groups on suturing the wounds, as perceived by patient, parent and staff. There was a significantly higher number of "failed" anaesthetics (pain scores 8-10) in the lignocaine group $(P<0 \cdot 01)$. On direct questioning the overall procedure was considered acceptable by $84.5 \%$ of parents in the AC group compared with $61 \%$ of parents in the lignocaine group $(P<0.01)$. There were no significant complications in either group. Conclusions-Topical AC should be considered the local anaesthetic of first choice for suturing appropriate children's lacerations.
\end{abstract}

(f Accid Emerg Med 1996;13:1 19-122)

Accident and Bristol Frenchay

Hospital

$\mathrm{J} M$ Kendall

Paediatric Trauma

Liaison,

Derbyshire Children's

Hospital/Royal

Infirmary

A Charters

Accident and

Emergency Medicine,

Gloucestershire Royal

Hospital

S E McCabe

Correspondence to:

J M Kendall MRCP

Accident and Emergency

Department,

Fepartment,

Frenchay Hospital,

Bristol BS16 1LE,
United Kingdom.

Key terms: adrenaline/cocaine; lignocaine; topical anaesthesia; children

Children are physically and psychologically different from adults, and the approach to the child in the accident and emergency (A\&E) department must be appropriately modified. Whether or not this is the child's first experience in a hospital environment, it is our responsibility to ensure it is as stress-free as possible.

It is no longer acceptable for children to be bundled into a blanket, held down, and sutured, leaving them with a fear of hospitals and nurses for many subsequent years. Legislation such as The Children's Act and
The Audit Commission Report gives children rights to informed consent and warns the clinician against what could be perceived as physical assault by nurses who force children into treatment that they don't fully understand.

Everyone who has worked in an $A \& E$ setting knows how distressing suturing children under local anaesthetic can be for the child, parents, and staff. It is not possible to explain the purpose of a local anaesthetic to a young frightened child; when conventional local anaesthesia is used, the sight of a needle, and the pain of infiltration of lignocaine, means that the child's confidence may be lost, even before the procedure to close the wound begins.

A topical preparation, which is "painless" to apply, of adrenaline $(1: 2000)$ and cocaine $(11.8 \%)$ is used extensively in the United States, Australia, and South Africa to locally anaesthetise children's lacerations before suturing them. ${ }^{1-3}$ There is little awareness of this preparation in the United Kingdom: a recent survey (with a $67 \%$ response rate) of 150 A\&E departments seeing over 30000 patients annually revealed that less than $5 \%$ of consultants had experience in the use of this preparation.

The rationale behind using this combination of ingredients is that the cocaine provides excellent local anaesthesia, while the combination of adrenaline and cocaine causes marked vasoconstriction, thus minimising bleeding and limiting systemic absorption of anaesthetic.

The objectives of our study were (1) to compare prospectively the anaesthetic properties of conventional intradermal $1 \%$ plain lignocaine with a topical gel preparation of adrenaline $(1: 2000)$ and cocaine $(4 \cdot 7 \%)$; and (2) to increase awareness of this preparation if its anaesthetic properties are found to be equal to lignocaine, and if its use is practical in $A \& E$ departments in this country.

\section{Methods}

SETTING

The study was carried out in the A\&E department of Gloucestershire Royal Hospital, a busy district general hospital seeing 40000 new attendance's per year, of which 10000 are children.

\section{SUBJECTS}

Children aged between 3 and 16 years consecutively presenting to the A\&E department from January to November 1994 with a laceration less than $4 \mathrm{~cm}$ in length anywhere on the body, excluding the digits and mucous membranes, were included in the trial. 
Previously published reports have shown that there are risks of cocaine toxicity if the adrenaline and cocaine (AC) preparation is used inappropriately on mucous membranes. ${ }^{3}$

PREPARATION OF ADRENAIINE AND COCAINE

There is no commercially available preparation of adrenaline and cocaine in the United Kingdom, so we provided the manufacturing department of our pharmacy with the pharmaceutical preparation sheet of the Royal Children's Hospital, Melbourne, Australia. They use a higher concentration of cocaine $(11.8 \%)$ in their preparation than we used for this trial $(4 \cdot 7 \%)$, and so the formula had to be modified appropriately. To ease application of the preparation, we made up the preparation in gel rather than liquid form. ${ }^{1}$

The gel is prepared by dissolving the cocaine powder $(1.58 \mathrm{~g})$ in a mixture of water for injection $\mathrm{BP}(14.5 \mathrm{ml})$ and adrenaline injection $1: 1000(15 \mathrm{ml})$. This solution is filtered through a 0.2 micron filter into an empty $50 \mathrm{ml}$ syringe. Presterilised Tylose powder $(1.65 \mathrm{~g})$ is added and mixing of the ingredients is aided by transferring the gel between two syringes through a sterile transfer device. The resulting gel is packed into Luer-lock syringes and capped. The whole process is carried out under aseptic conditions to ensure a clean product.

The syringes are stored at room temperature, using appropriate precautions for a controlled drug. A complete manufacturing worksheet for the preparation and storage of AC is available from the Gloucester Royal NHS Trust Pharmacy Production Unit.

\section{PROCEDURES}

Children presenting with an appropriate laceration were consecutively assigned to receive either conventional intradermal lignocaine, or topical AC preparation. The parents gave informed consent for their child to be entered into the trial. Ethical approval was received from the ethics committee of Gloucester Royal Hospital.

The nature of the trial meant that the two groups could not be blinded.

Plain lignocaine $(1 \%)$ was infiltrated locally in conventional manner using a syringe and needle; the method of infiltration was stan-

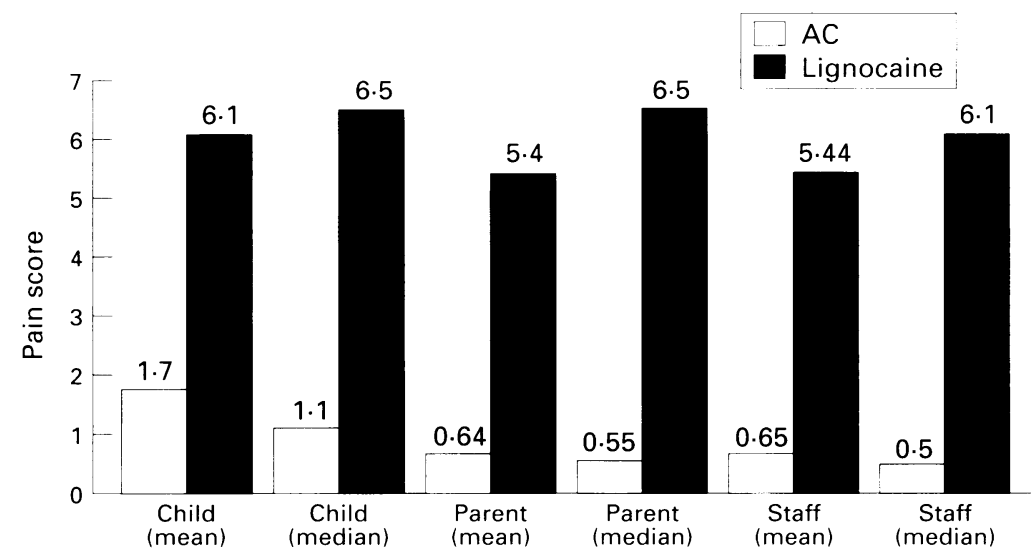

Figure 1 Pain scores on administration of anaesthetic. dardised using a 25 gauge needle. The lignocaine was at room temperature and was not buffered.

AC preparation was given as follows: $1 \mathrm{ml}$ of AC to $1 \mathrm{~cm}$ laceration; half of the gel was applied directly to the wound, the rest was applied to a gauze swab laid on the wound surface. The swab was left in place for 10-15 min, observing for blanching of the surrounding skin caused by local vasoconstriction due to the adrenaline.

The wounds were sutured in a conventional manner. Needle gauge and suture size and material were the same for both groups.

Perception of pain by child, parent, and staff was recorded during infiltration or application of anaesthesia and during wound closure. Pain scoring followed conventional techniques using Wong Baker faces for children under the age of 10, and visual analogue scores for older children, parents, and staff. ${ }^{4}$

Overall acceptability of the procedure was assessed by direct questioning of the parents at the time of discharge and on follow up.

All of the children were followed up at 5-7 d for removal of sutures and assessment of any complications, and for any feedback from the parents.

We used $\chi^{2}$ and Mann Whitney $U$ tests for statistical analysis.

\section{Results}

The total number of children who presented with lacerations during the study period was 1290; of these, $230(18 \%)$ subsequently needed sutures (the majority being stapled, glued, or repaired with Steristrip). One hundred and twenty $(53 \%)$ had lacerations that were appropriate for $\mathrm{AC}$ (the rest occurring on, or very near, mucous membranes, or on the extremities). These 120 children with appropriate lacerations were recruited into the study; 13 were subsequently excluded for the following reasons: eight because of incomplete data collection, two because they received Steristrips instead of sutures after local anaesthesia, and three who did not attend follow up.

There were no significant differences between the two groups in terms of age of child, or the length or location of the laceration (table 1).

Mean and median pain scores on administration of anaesthetic and on wound closure (table 2) are shown in figs 1 and 2.

Mean and median pain scores for the infiltration of lignocaine were significantly higher than for the application of $\mathrm{AC}$ as perceived by child, parent, and staff $(\mathrm{P}<0.001)$. There was no significant difference in the mean and median pain scores between the two groups on wound closure.

Table 1 Patient details

\begin{tabular}{lcc}
\hline & $\begin{array}{c}A C \\
(n=56)\end{array}$ & $\begin{array}{l}\text { Lignocaine } \\
(n=51)\end{array}$ \\
\hline Mean age (years) & $7 \cdot 10$ & $7 \cdot 35$ \\
Mean length of laceration $(\mathrm{cm})$ & 1.96 & 1.90 \\
Laceration on trunk or limbs & 23 & 22 \\
Laceration on face or scalp & 33 & 29 \\
\hline
\end{tabular}

$\mathrm{AC}$, adrenaline plus cocaine. 
Table 2 Mean and median pain scores

\begin{tabular}{lllllll}
\hline & & \multicolumn{2}{l}{$\begin{array}{l}\text { Pain score, } \\
\text { AC group }\end{array}$} & & \multicolumn{2}{l}{$\begin{array}{l}\text { Pain score, } \\
\text { lignocaine group }\end{array}$} \\
\cline { 3 - 4 } & & Mean & Median & & Mean & Median \\
\hline Administration of local anaesthetic & Child & $1 \cdot 70$ & $1 \cdot 10$ & & $6 \cdot 10$ & $6 \cdot 50$ \\
& Parent & $0 \cdot 64$ & $0 \cdot 55$ & & $5 \cdot 40$ & $6 \cdot 50$ \\
& Staff & $0 \cdot 65$ & $0 \cdot 50$ & & $5 \cdot 44$ & $6 \cdot 10$ \\
& Child & $4 \cdot 50$ & $4 \cdot 60$ & & $4 \cdot 40$ & $4 \cdot 30$ \\
& Parent & $3 \cdot 10$ & $3 \cdot 20$ & & 3.80 & $3 \cdot 50$ \\
& Staff & $2 \cdot 60$ & $2 \cdot 50$ & & 3.60 & $3 \cdot 10$ \\
\hline
\end{tabular}

AC, adrenaline plus cocaine.

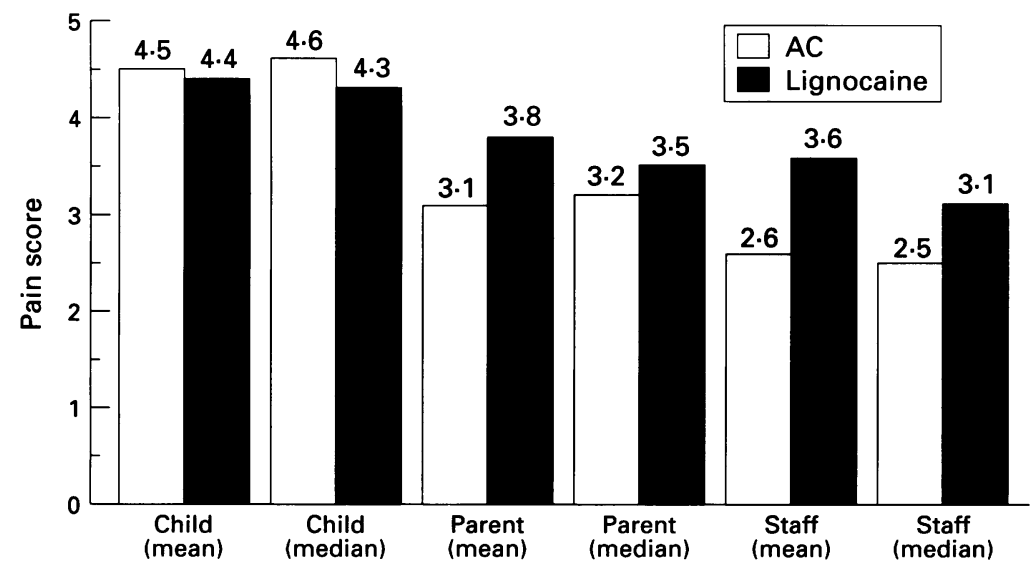

Figure 2 Pain scores on wound closure.

Fifty seven per cent of all documented pain scores on wound closure in the AC group were between 0 and 3 inclusive, compared with $55 \%$ in the lignocaine group (NS); this pain score bracket was considered to reflect "adequate" anaesthesia (table 3, fig 3).

Ten per cent of all documented pain scores on wound closure in the AC group were between 8 and 10 inclusive, compared with $24 \%$ in the lignocaine group $(P<0.01)$; this pain score bracket was considered to reflect "failed" anaesthesia.

In the AC group, $14.5 \%$ of parents considered the procedure as a whole to be

Table 3 Overall pain scores on wound closure

\begin{tabular}{lll}
\hline & \multicolumn{2}{l}{ Child, parent, and staff scores } \\
\cline { 2 - 3 } & $\begin{array}{l}A C, \\
n(\%)\end{array}$ & $\begin{array}{l}\text { Lignocaine, } \\
n(\%)\end{array}$ \\
\hline Pain score 0-3, "adequate" & $95(57)$ & $84(55)$ \\
Pain score 8-10, "Failed" & $17(10)$ & $37(24)$ \\
\hline
\end{tabular}

AC, adrenaline plus cocaine.

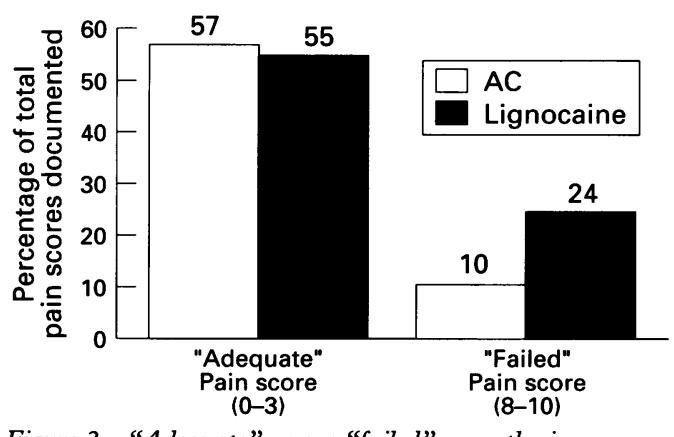

Figure 3 "Adequate" versus "failed" anaesthesia. "stressful" or "unacceptable", compared with $39 \%$ in the lignocaine group $(P<0.01)$.

\section{Discussion}

The application of the topical AC preparation was considerably less painful than the local infiltration of lignocaine (marked statistical significance was reached for child, parent, and staff pain perception). This shows the considerable benefit of the topical preparation, provided its anaesthetic qualities are equivalent or superior to lignocaine on wound closure.

No significant differences were found in the mean or median pain scores between the two groups on wound closure. It is apparent that whichever method of local anaesthesia we used, there is no such thing as "painless" anaesthesia during a stressful procedure on a child. Visual analogue pain scoring has limitations but is recognised as being the most reliable method of pain assessment. ${ }^{4}$ It is easier and more reliable for a parent or staff member than for a child to distinguish the pain of a particular event (for example, suture insertion, lignocaine infiltration, etc) from fear of the overall procedure. Pain scoring by the child alone will be influenced by other factors such as the fear of being in an unusual environment with unrecognised people; pain scoring is therefore more reliable with more than one observer.

An alternative, perhaps more useful, way of comparing AC with lignocaine is to determine the total number of "adequate" and "failed" anaesthetic episodes, as reflected in the pain scores on wound closure in the two groups. The pain score bracket $0-3$ was arbitrarily considered to reflect "adequate" anaesthesia, and the bracket 8-10 "failed" anaesthesia. There was no significant difference (table 3; fig 3) between the proportion of adequate anaesthetics in the AC group (57\%) and the lignocaine group $(55 \%)$. There was, however, a significantly higher proportion of failed anaesthetics in the lignocaine group compared with the AC group $(24 \% v 10 \%, P<0.01)$. This difference in failure rate is also reflected on direct questioning of the parents, of whom $14.5 \%$ considered the overall procedure to be stressful or unacceptable in the AC group, compared with $39 \%$ in the lignocaine group $(\mathrm{P}<0.01)$.

Complication rates in both groups were low; this is in accordance with published reports. ${ }^{24}$ One patient in the lignocaine group had a small area of wound dehiscence which closed uneventfully by secondary intention. One patient in the AC group had a small haematoma which developed under the wound which settled spontaneously, and a second patient had a small area of erythema surrounding the wound at follow up, which resolved with flucloxacillin.

In the USA and Australia, higher concentrations of cocaine $(11.8 \%)$ are used in the preparation; we could only get ethical approval to use $4 \cdot 7 \%$ cocaine because of the possibility of systemic absorption of cocaine and potential toxicity. When used appropriately, published reports suggest that, while absorption does occur, producing low but measurable amounts of cocaine metabolites, ${ }^{56}$ there is no clinical $^{6} 7$ 
or systemic ${ }^{8}$ toxicity; there are rare cases reported of seizures and even deaths occurring when the preparation has been used inappropriately on mucous membranes. ${ }^{9}$ Further studies are now ongoing using a higher concentration of cocaine in a blinded trial to evaluate if we can improve its anaesthetic efficacy.

The AC preparation is a controlled drug and the procedures for the storage, prescription, and administration of a controlled drug need to be employed; this did not present any practical difficulties in its day to day usage. There is potential for theft and abuse, as for any controlled drug.

Patient flow is an important factor in an $A \& E$ department and therefore duration of the procedure needs to be considered: the application of AC takes less time than the infiltration of lignocaine, and the onset of anaesthesia is comparable (10-15 min). There are no significant time implications in its use.

Cost needs to be considered; the price of a single application of $\mathrm{AC}$ at the present time is approximately $£ 2$; it is not commercially available and is prepared in small quantities by our pharmacy, who estimate that the cost could be much less if produced in larger amounts. Therefore the price per patient is more than lignocaine (which is approximately $25 \mathrm{p}$ for a $10 \mathrm{ml}$ vial of $1 \%$ concentration); considering patient volume, the overall cost implications are tiny, and individual patient benefit is considerable.

\section{Conclusions}

A topical preparation of adrenaline and cocaine is just as effective in its local anaesthetic prop- erties as intradermal lignocaine in the suturing of children's lacerations, and is significantly less painful to apply. Its use is associated with a lower anaesthetic "failure" rate and significantly higher parental acceptability for the overall procedure of wound closure.

We encountered no significant complications with the use of AC, and this is compatible with the published reports and its widespread use in other countries. It is a practical alternative to lignocaine and should be considered as the local anaesthetic of choice in children with lacerations of less than $4 \mathrm{~cm}$ length not involving the digits or mucous membranes.

1 Bonadio WA, Wagner VR. Adrenaline-cocaine gel topical naesthetic for dermal laceration repair in children. $A n n$ Emerg Med 1992;21:1435-8.

2 Bonadio WA, Wagner VR. Efficacy of tetracaine adrenaline cocaine topical anaesthetic without tetracaine for facia laceration repair in children. Pediatrics 1990;86:856-7.

3 Anderson AB, Colecchi C, Baronoski R, DeWitt TG. Local anaesthesia in paediatric patients: topical TAC versus lidocaine. Ann Emerg Med 1990;19:63-7.

4 Huskisson EC. Measurement of pain. Lancet 1974;ii: $1127-31$

5 Smith SM, Barry RC. A comparison of three formulations of TAC (tetracaine, adrenaline, cocaine) for anaesthesia of minor lacerations in children. Pediatr Emerg Care 1990;6:266-70.

6 Fitzmaurice LS, Wasserman GS, Knapp JF, Roberts DK, Waekerle JF, Fox M. TAC use and the absorption of cocaine in a paediatric emergency department. Ann Emerg Med 1990;19:515-8.

7 Bass DH, Wormald PJ, McNally J, Rode H. Topical anaesthesia for the repair of minor lacerations. Arch Dis anaesthesia for the repair

8 Hegenbarth MA, Altieri MF, Hawk WH, Greene A Ochsenschlager D, O'Donnell R Comparison of topical techsenschlager $\mathrm{D}$, $\mathrm{O}$, Donnell $\mathrm{R}$ Comparison of topical lidocaine infiltration for repair of lacerations in children lidocaine infiltration for repair

9 Foley J. TAC: a controversial topical anaesthetic for suture of skin lacerations. 7 Emerg Nurs 1994;20:221-2. 